\title{
ASSESSMENT OF THE MICROSCOPIC- OBSERVATION- DRUG - SUSCEPTIBLITY ASSAY (MODS) AND GENEXPERT ASSAY FOR DIAGNOSIS OF PULMONARY TUBERCULOSIS
}

\author{
By
Eman Hosny Mohamad, Effat Mostafa Hasan, Faten Aly Shoukry* and Omnia Ahmed El-Dydamoni
Department of Microbiology, Al-Azher Faculty of Medicine (Girls) and National TB Program*

Corresponding author email: Emanhosny22@yahoo.com,

Tel: 01226883232

\begin{abstract}
Background: Tuberculosis (TB) is considered the most common and highly contagious disease worldwide. Early and accurate diagnosis of TB with detection of multidrug-resistant Mycobacterium tuberculosis (M.TB) is of primary importance for both patient management and infection control.

Objectives: To evaluate the performance of Microscopic-Observation Drug-Susceptibility (MODS) assay and GeneXpert MTB/RIF( GeneXpert ) assay in diagnosis of TB and detection of MDR-TB directly from sputum specimens in comparison to the convential diagnostic methods (direct Ziehl-Neelsen "Z-N" stained smear and culture on Lowenstein-Jenson media "LJ").

Patients and Methods: After approval of institutional ethical committee a total of 120 suspected pulmonary TB patients admitted to chest hospitals and dispensaries in Egypt (Giza, Cairo, Alexandria, Dakahlia, Suez, Beheira, Monufia, Qena). Their age was more than 16 years; all of them did not receive any anti tubercular drugs. Sputum samples were collected from each patient and subjected to direct ZN smear, culture and sensitivity of M.TB on LJ and Middlebrook 7H9 medium for MODS assay. Finally Genexpert M.TB/RIF assay was done directly for all sputum samples.

Results: Infection rate among males was higher than female $(81.7 \%$ \& $18.7 \%)$. Also, it was observed that $56.7 \%$ of sputum samples gave positive direct Z-N smear, while $64.2 \%$ were positive for both $\mathrm{LJ}$ culture \&MODS assay. On the other hand, by Genexpert, $68 \%$ of cases were positive. The sensitivity and specificity of the various diagnostic methods were studied in relation to LJ culture (gold standard). It was found that MODS assay was rapid that results were obtained in a median of 8 days ranged from (7- 21 days), while median time of LJ was 21dayes ranged from (21-60). MODS had 100\% sensitivity and specificity. Direct smear was less sensitive $(88.3 \%$ ), and GeneXpert was less specific $(88.4 \%)$, but more rapid results were obtained in 2 hours. Finally, detection of MDR-TB using various methods for 77 isolated M.TB strains ranged from $16.9-19.5 \%$ to isoniazid (INH) and 9.8\%-18.2\% to rifampicin (RMP) by various diagnostic methods with statistically insignificant difference between them.

Conclusion: MODS was an optimal alternative rapid diagnostic technique for TB and detection of M.TB and MDR-TB in resource limited settings, results were obtained in a median of 8 days. On the other hand, Gene Xpert was a novel integrated diagnostic PCR analysis in a single hand free step for rapid diagnosis of $\mathrm{TB}$ and detection of RMP resistance in clinical specimens in 2 hours but it was expensive.
\end{abstract}

Key words: MODS, GeneXpert, M.TB, MDR-TB, TB, and PCR. 


\section{INTRODUCTION}

Although much progress has been made in improving TB cure rates, diagnosis and case detection remain major obstacles to TB control. Conventional drug susceptibility testing (DST) methods for M. TB take approximately 6 weeks to yield results, which leads to loss of time and delayed initiation of proper treatment, resulting in the transmission of drug resistant strain in the community (Pai et al., 2009). Advances in technology have led to the development of newer modalities of DST based on automated systems or molecular diagnostic methods. These tests, however, are expensive. In resource-poor settings, a rapid, reliable yet inexpensive DST method is urgently required. The application of rapid, sensitive and inexpensive diagnostic tests such as the microscopic-observation drugsusceptibility MODS is capable of identifying MDR-TB aids in the early initiation of treatment and prevents further transmission (WHO, 2017). MODS assay is a phenotypic rapid diagnostic test for TB and testing for at least INH and RMP resistance (Sanogo et al., 2017). Rapid identification, which is essential for earlier treatment initiation, improved patient outcomes, and more effective public health interventions, relies on nucleic acid amplification techniques. The GeneXpert MTB/RIF assay is a novel integrated diagnostic device that performs sample processing and hemi nested real-time PCR analysis in a single hands-free step for the diagnosis of tuberculosis and rapid detection of RMP resistance in clinical specimens in two hours (Wilson, 2014 and Boehme \& Nabeta, 2014). The information provided by the GeneXpert aids in selecting treatment regimens and reaching infection control decisions quickly (Mohamed et al., 2019).

\section{PATIENTS AND METHODS}

This work was conducted on 120 suspected patients of pulmonary tuberculosis. They were chosen according to the clinical manifestation and radiological data. These cases were selected from chest hospitals in Egypt and dispensary (Giza, Cairo, Alexandria, Dakahlia, Suez, Beheira, Monufia and Qena) from January 2016 to June 2019. Their ages were more than 16 years, (16 females and 104 males). All patients were complaining with prolonged night fever, fatigue, loss of weight, bloody sputum and positive radiological data. They did not receive any anti- tuberculous drugs. The collected sputum samples were processed in the central laboratories of Egypt.

Three sputum samples were collected from each patient in $50 \mathrm{ml}$. screw caped plastic sterile container. Each patient sample was subjected for direct Z-N smear, then culture and sensitivity of M.TB on L-J medium and Middlebrook 7H9 medium for MODS assay after sputum decontamination and processing. One $\mathrm{ml}$ of sputum sample was used for GeneXpert M.TB/RIF assay.

\section{The following laboratory techniques were used :}

I. Smear staining by Z-N stain technique: was described by Kent and Kubica (1985).

II. Sputum decontamination and processing: Sputum decontamination is performed with the sodium hydroxide$\mathrm{N}$-Acetyl-L-Cysteine (NaOH-NALC) 
method was described by Kent and Kubica. (1985).

III. Culture and sensitivity of M.TB on LJ medium: Was done as Canetti methods (Canetti et al., 1969).

IV. Culture and sensitivity of M.TB on (MODS) assay: MODS assay was a liquid culture based test that detects M.TB and assessed (INH) and (RMP) susceptibility directly from sputum samples. This was done regarding the MODS user guide (2008).

Twenty four well plates were inoculated with decontaminated sputum samples suspended in Middle brook $7 \mathrm{H} 9$ medium then examined for micro colonies which was detected in a medium after 7 days. A characteristic microscopic cording appeared of that growth in liquid media using an inverted light microscope. The growth was usually much earlier than macroscopic colony growth that can be seen on solid medium. The incorporation of isoniazid and rifampicin in the testing process enabled equally rapid MDR -TB detection. This method was characterized by the growth in liquid media is markedly faster than on solid media, an easily recognizable M.TB growth, and rapid detection of MDR strain of M.TB. This was done regarding the MODS user guide (2008).
V-GeneXpert MTB/RIF assay: The GeneXpert Dx System integrates and automates sample processing, nucleic acid amplification, and detection of the target sequences in samples using real-time PCR (Helb et al., 2010).

\section{Statistical analysis:}

1. Coding of data was carried out manually, and analysis was conducted through SPSS program version 15 (SPSS Inc., Chicago Ill, 2006).

2. Descriptive and analytical statistical analyses were done.

\section{Descriptive statistics:}

- Quantitative data: mean and standard deviation "SD" were used as they measure central tendency and dispersion of quantitative data.

- Qualitative data were expressed in numbers and by using test of significance as: Chi square-test $(\chi 2)$ : for comparison of percentage.

The study was reviewed and approved by the Research Ethics committee at the AL- Azhar University, Faculty of Medicine for Girls and by REC decision of the Ministry of Health and Population at the Central Directorate of Research and Health Development \& Review.

\section{RESULTS}

A total of 120 suspected patients of pulmonary tuberculosis were selected and sent the collected sputum samples to Central Laboratories of Egypt from January 2016 to June 2019. Their ages were more than 16 years, (16 females and 104 males) (Table 1). It was demonstrated that $81.7 \%$ of patients were males ( $\mathrm{p}$ value
$<$ 0.001). According to geographic distribution, Giza, Cairo \& Dakahlia proved more frequent rates of TB infection (30, 27.5, and 17.5, respectively).

Comparison between the various diagnostic tests of tuberculosis in relation to culture on L-J medium (Figure 1). We 
found that the results of direct stained smears were the less than culture on L-J and MODS (56.7, 64.2, and 64.2), respectively. It was noticed that the results of MODS culture were as similar as L-J culture but it was more rapid (require 7-21 days incubation). On the other hand Gene Xpert gave higher results $(68.3 \%)$, in which 82 sputum samples were positive. However five sputum samples which were negative using L-J medium and MODS culture proved positive results by Gene Xpert assay (Figure 1).

The sensitivity, specificity and accuracy of direct Z-N smear, MODS and Gene Xpert were studied in comparison to culture on LJ (gold standard). It can notice the sensitivity, specificity \& accuracy of MODS was $100 \%$, while direct smear was less sensitive $88.3 \%$. Also, GeneXpert showed $100 \%$, sensitivity but specificity $(88.4 \%)$ and accuracy $95.8 \%$ (Table 2 and Figure 2).

Moreover, the drug sensitivity test of M.TB isolates to INH using L-J medium and MODS assay (Table 3). The results showed a statistically insignificant difference between both methods ( $\mathrm{P}$ value > 0.6). Also, drug sensitivity test of M.TB to RMP using culture on L-J, MODS and Gene Xpert (Table 4). The results diagnosed statistical insignificance difference between the various methods ( $\mathrm{P}$ value $>0.4)$.

Table (1): Demographic data of 120 suspected tuberculous patients in relation to gender and geographical distribution.

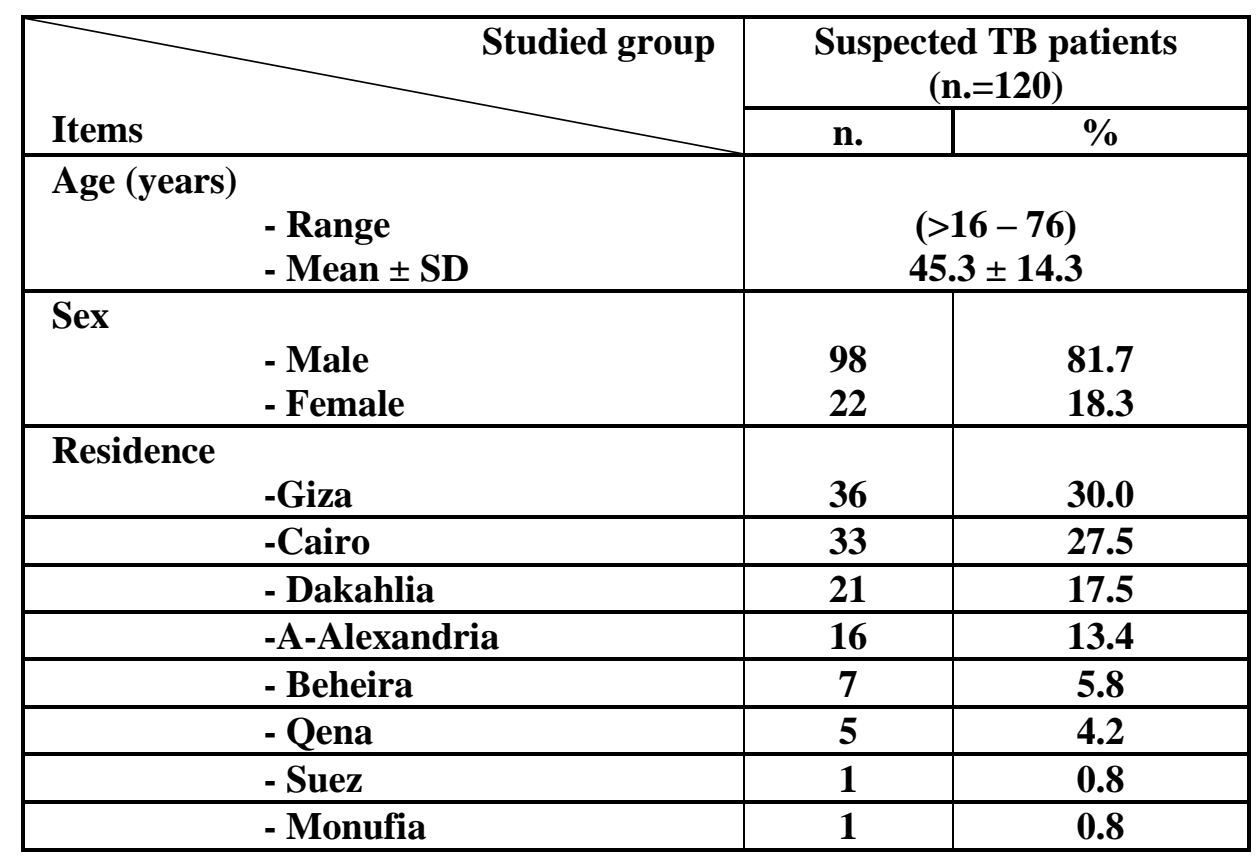




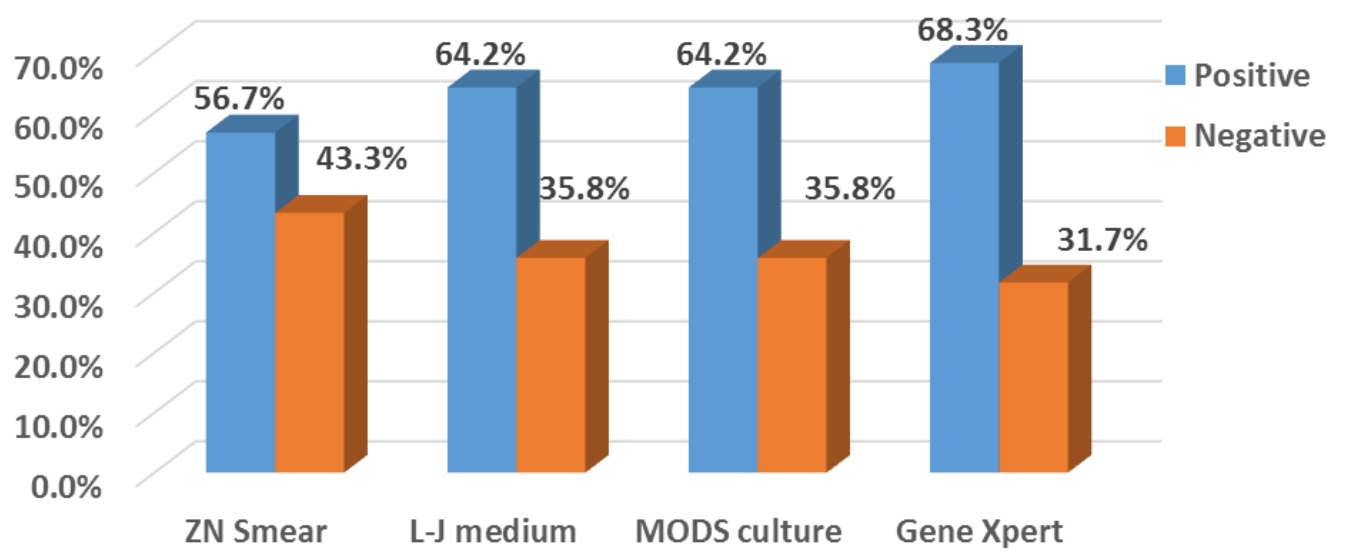

Figure (1): The results of four diagnostic tests used for 120 suspected pulmonary TB

Table (2): Comparison between the various diagnostic methods of tuberculosis in relation to culture on $\mathrm{L}-\mathrm{J}$ medium (the gold standard)

\begin{tabular}{|c|c|c|c|c|c|}
\hline \multirow{2}{*}{ Studied groups } & \multicolumn{4}{|c|}{ Culture on L-J medium } & \multirow{2}{*}{ p.value } \\
\cline { 2 - 5 } & Positive (77) & \multicolumn{2}{c|}{ Negative (43) } & \\
\cline { 2 - 5 } & No. & $\%$ & No. & $\%$ & \\
\hline ZN Smear & & & & & \\
$+\mathrm{Ve}$ & 68 & 88.3 & 0 & 0.0 & \\
$-\mathrm{Ve}$ & 9 & 11.7 & 43 & 100.0 & 0.001 \\
\hline $\begin{array}{c}\text { Culture on MODS } \\
+\mathrm{Ve}\end{array}$ & 77 & 100.0 & 0 & 0.0 & \\
$-\mathrm{Ve}$ & 0 & 0.0 & 43 & 100.0 & \multirow{2}{*}{0.001} \\
\hline $\begin{array}{c}\text { Gene Xpert } \\
+\mathrm{Ve}\end{array}$ & 77 & 100.0 & 5 & 11.6 & \\
$-\mathrm{Ve}$ & 0 & 0.0 & 38 & 88.4 & 0.001 \\
\hline
\end{tabular}




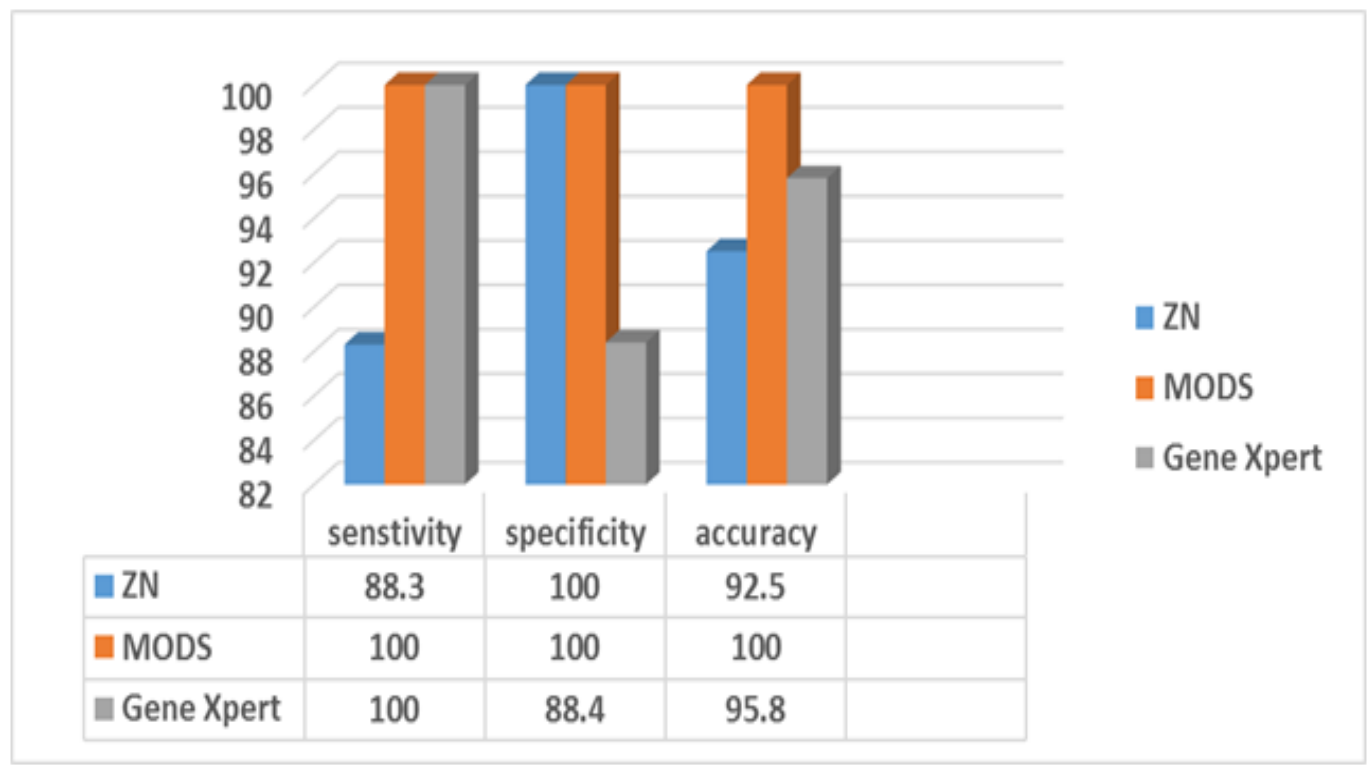

Figure (2): The sensitivity, specificity and accuracy of the various diagnostic methods of TB in relation to culture on $\mathrm{L}-\mathrm{J}$ medium (the gold standard)

Table (3): Comparative study drug sensitivity test of 77 M.TB isolates to INH using L-J medium and MODS

\begin{tabular}{|l|c|c|c|c|c|}
\hline \multirow{2}{*}{ INH } & \multicolumn{2}{|c|}{ Sensitive } & \multicolumn{2}{c|}{ Resistant } & \multirow{2}{*}{ p-value } \\
\cline { 2 - 5 } & No. & $\%$ & No. & $\%$ & \multirow{2}{*}{0.676} \\
\hline Culture on L-J medium & 64 & 83.1 & 13 & 16.9 & \multirow{2}{*}{0.676} \\
\hline Culture on MODS & 62 & 80.5 & 15 & 19.5 & \\
\hline
\end{tabular}

Table (4): Results drug sensitivity test of M.TB isolates to RMP using L-J medium, MODS and GeneXpert

\begin{tabular}{|l|c|c|c|c|c|}
\hline \multirow{2}{*}{ RMP } & \multicolumn{2}{|c|}{ Sensitive } & \multicolumn{2}{c|}{ Resistant } & \multirow{2}{*}{ p-value } \\
\cline { 2 - 5 } & No. & $\%$ & No. & $\%$ & \multirow{2}{*}{0.296} \\
\cline { 1 - 5 } Culture on L-J medium & 63 & 81.8 & 14 & 18.2 & \multirow{2}{*}{} \\
\hline MODS assay & 67 & 87.0 & 10 & 13.0 & \\
\hline Gene Xpert & 74 & 90.2 & 8 & 9.8 & \\
\hline
\end{tabular}

\section{DISCUSSION}

This work was carried out on 120 suspected patients of pulmonary tuberculosis. They were chosen according to the clinical manifestation and radiological data. The patients didn't receive anti- tuberculous drugs. These patients were selected from some chest hospitals of Egypt and dispensary. It was observed that nearly three quarters of new cases, were males with statistically significant difference. These result was in agreement with the study done by $W H O$ (2018) reported that males exceed females TB infection in Egypt due to sociocultural factors. Men have more social 
contacts and thus run a greater risk of exposure to contagious patient. Also exposure to predisposing factors like smoking, alcohol intake and drug abuse have considerable effects. More over immunological differences between men and women make males more susceptible than females to some infections (Heba, 2014).

In our study, comparison between the various diagnostic tests was presented. It was found the direct stained smear was the less sensitive than culture on L-J and MODS; however the result of GeneXpert gave higher percentage (68.3\%).

It can be noticed that $\mathrm{Z}-\mathrm{N}$ smear was cheap, rapid and widely used methods in all laboratories allover Egypt that with sensitivity (88.3\%), specificity (100.0\%), and accuracy (92.5\%) compared with culture on L-J medium .

Our results were quite similar to that study done by Husseiny et al. (2016) from Egypt who reported that sensitivity $(86.4 \%)$ and excellent specificity $(99.7 \%)$ for Z-N. These results were also quite similar to those obtained by Bunsow et al. (2014) where Z-N showed sensitivity of $(78.5 \%)$ and specificity of $(98.3 \%)$ when compared with L-J culture as gold standard. In other study done by Blakemore et al. (2010) who compared acid fast bacilli (AFB) detection for diagnosis of $\mathrm{TB}$ by $\mathrm{Z}-\mathrm{N}$ in relation to culture on L-J medium. It showed that (98\%) sensitive and (48\%) specific for smear-positive.

In this result, sensitivity of MODS was $100.0 \%$, specificity $100.0 \%$, and accuracy $100 \%$, with median time 8 days for the detection of tubercle bacilli compared with the culture on L-J medium, while median time of L-J was 21dayes. Huang et al. (2015) carried out a prospective study on MODS in 5 laboratories situated in China and found that sensitivity of MODS ranged between $87.78 \%$ and $94.4 \%$ and specificity between $96.8 \%$ and $100 \%$.

Sanogo et al. (2017) found that the specificity and sensitivity of MODS assay to diagnose tuberculosis infection were determined in their population to be $88.73 \%$ and $88.24 \%$, respectively; in positive sputum smears, naive to $\mathrm{TB}$ treatment. Also the result that obtained in Egypt by Rasslan et al. (2012) reported that, the sensitivity, specificity of MODS in the detection of M.TB were respectively $95.3 \%$ and $98.6 \%$. MODS results were obtained in a median of 8 days while L-J were obtained in a median 21days.

The important of MODS elevated in most previous studies including ours because M.TB grows faster in liquid medium than in solid medium plus an inverted microscope is used to detect the cording, which is characteristic of M.TB in liquid medium but L-J culture detection of the colonies by naked eyes.

This was agreed with the study done by Rasslan et al. (2012) from Egypt they found that MODS assay is a rapid, economical, and highly sensitive and specific method for the detection of M.TB and DST directly from a sputum samples, results were obtained in a median of 8 days, but they were compared their result to BACTEC MGIT 960 and they were only examined known smear-positive patients, but in our study investigated a suspected TB patients and L-J was gold sundered. 
The current study revealed that the sensitivity of the GeneXpert compared with the culture on L-J positive specimens was $(100.0 \%)$, specificity $(88.4 \%)$, and accuracy $(95.8 \%)$. Near result was observed by Mohamed, et al. (2019) in Egypt who found that GeneXpert test has 97.6\% sensitivity and $81 \%$ specificity, while Iram et al. (2015) from Pakistan, who reported $100 \%$ sensitivity and $86 \%$ specificity for Gene Xpert.

Husseiny et al. (2016) reported that the sensitivity and specificity of the GeneXpert assay were $93 \%$ and $98.3 \%$ respectively. Monika et al. (2016) found that the sensitivity and specificity of GeneXpert in sputum assay was (100\%) and $(90 \%)$. Other study of Sharma et al. (2015) who reported that sensitivity and specificity were $96.9 \%$ and $99.8 \%$ in this subject.

Our study found that GeneXpert detects 5 positive sputum samples which were -ve by L-J culture and MODS, Thus, the Gene Xpert assay has a sensitivity that approximately approaches that of culture. This is in agreement with Bodmer and Ströhle,(2012) who stated that the Gene Xpert overall clinical sensitivity of detecting M.TB in sputum of patients with suspected pulmonary TB when compared to L-J culture as the reference, this can be explained by death of many M.TB during processing and decontamination process of sputum samples while GeneXpert can detect both live and dead bacteria.

Finally, detection of MDR-TB using various methods for 77 isolated M.TB strains was ranged from $16.9 \%-19.5 \%$ to INH and $9.8 \%-18.2 \%$ to RMB by various diagnostic methods with statistically insignificant difference between them ( $p$ value > 0.4 ).

This was similar to WHO (2017) who reported that MDR TB in Egypt increased that was reach $14 \%$ in 2016.

In comparison of various methods for diagnosis INH resistant, it was found that $19.5 \%$ (15) cases resistant and 2 cases mono resistant to INH on MODS while DST on L-J media detect $16.9 \%$ (13) cases, suggests that culture on MODS for diagnosis INH resistant was best for diagnoses. This in agree with Rasslan et al. (2012) found that the ability to detect MODS susceptibility for INH was more than RMP .

Sanogo et al. (2017) showed that MODS techniques showed identical mono-resistance to INH of $6.15 \%$, this confirm that MODS more sensitive for detection of INH resistant more than RMP .

In our study, the comparison between the three tests in diagnoses of RMP resistant we noticed that in our result, L-J detect 14 cases resistant to RMP .

There were 6 cases only resistant to RMP on L-J media and 2 of them only resistant to RMP on MODS culture while GeneXpert detected 8 cases only it suggest that there is a mutation outside the M. TB rpoB gens (GeneXpert are specific only to rpoB gens) suggests that it may be resistant outside rpoB gene because Gene Xpert detect only 8 cases .

Sanchez (2015) found that GeneXpert was designed to identify RIF-resistance mutations in an 81-bp region (codons 426 to 452 ) of the rpo- $\beta$ gene known as the RIF-resistance-determining region (RRDR). 
The accuracy for identification of RMP resistance was $98 \%$. However, a study done in Swaziland demonstrated that the assay may not be able to detect wild type mutations for RIF-resistance outside the rpo- $\beta$ I491F domain (Lawn et al., 2013).

This may clarify the difference in the results between the conventional and the GeneXpert method. In addition, this result can also be clarified by Lawn and Nickol (2011) who reported that to enable detection of rifampicin resistance by the Xpert, there must be present between 65 and $100 \%$ of the DNA from the RMPresistant isolate depending on the mutation. They suggested that in patients with mixed infections, the GeneXpert assay might only detect the resistant strain if it was the predominant one present.

GeneXpert also has a limited ability to identify some RMP resistance. WHO (2018) recommended Cepheid GeneXpert ( $)$ MTB/RIF Ultra cartridge (hereinafter Ultra MTB/RIF) that can both diagnose M.TB and indicate MDR- TB that more sensitive and specific.

\section{CONCLUSION}

MODS assay is a rapid, economical, and highly sensitive and specific method for the detection of M.TB and DST directly from a sputum sample and within 8 Days in comparable to L-J culture of M.TB and DST that take more time for its detection. Conventional DST takes more than 60 days to confirm the MDR in contrast to GeneXpert assay which is an extremely helpful diagnostic tool for the detection of rifampicin resistance in tuberculosis. Moreover GeneXpert assay is fairly highly sensitive and specific along with short turnout time in 2 hours but it is very expensive.

\section{REFERENCES}

1. Blakemore R, Story E, Helb D, Kop J, Banada P, Owens MR, Chakravorty $S$, Jones $M$ and Alland D. (2010): Evaluation of the analytical performance of the Gene Xpert MTB/RIF assay. Clin. Micro. J., 48: 2495-2501.

2. Bodmer $T$ and Ströhle A. (2012): Diagnosing Pulmonary Tuberculosis with the Gene Xpert MTB/RIF assay. Journal of Vis. Exp. j., 62: $3547-3553$.

3. Boehme C C and Nabeta P. (2014): Rapid molecular detection of tuberculosis and rifampin resistance. N. Engl. Med. J., 363: $1005-1015$

4. Bunsow E, Ruiz-Serrano MJ, Lopez Roa P, Kestler M, ViedmaDG and Bouza E. (2014): Evaluation of GeneXpert MTB/RIF for the detection of MT.B and resistance to rifampin in clinical specimens.Infect. J., 68: $338-343$

5. Canetti G. Fox W, Khousenko A. Mahler H T, Menon N K, Mitchison D A, Rist N and Surden NA. (1969): Advances in techniques of testing mycobacterial drug sensitivity, and the use of sensitivity tests in tuberculosis control programmes. Bull. W. H. O., 41: 21- 43 .

6. Caviedes L, Lee TS, Gilman RH, Sheen P, Spellman E, Lee EH, Berg DE and Montenegro James S. (2000): Rapid, efficient detection and drug susceptibility testing of M.TB in sputum by microscopic observation of broth cultures.Clin. Micro. J., 38 (3):1203-1208.

7. Heba M A. (2014): Quality of life among pulmonary tuberculosis patients, Thesis Submitted For Partial Fulfillment of M.D Degree in Microbiology, pp 138-140.

8. Helb D, Jones M, Story E, Boehme C, Wallace E, Ho K, Kop J, Owens MR, Rodgers R, Banada P, Safi $H$ and Blakemore R. (2010): Rapid detection of M.TB and rifampin resistance by use of ondemand, near-patient technology. Clin. Micro. J., 48: 229 -237. 
9. Huang $Z$, QinC, Jinghui $D$, Qing $L$, Yaoxing W, Wang Z, ZhangX, Guoliang $X$,Jie $C$, Xiaomeng $X$, Weiting $L$ and Junming L. (2015) : Evaluation of the MODS for the rapid detection of MDR-TB and XDR-TB in China: aprospective multicentre study. Antimicrob. Chemother. J., 70 (2):456 - 462.

10. Husseiny $S$ M, Faten $S$ B and Ahmed MA. (2016): Evaluation of GeneXpert MTB/RIF assay for direct diagnosis of pulmonary tuberculosis,Saudi. Med. J., 37 (10): 1076 1081.

11. Iram S, Hussain S. Zeenat A, Yusuf NW and Aslam M. (2015): Rapid diagnosis of tuberculosis using Xpert MTB/RIF assayreport from a developing country,Med. Sci.Pak. J., 31 (1): 105-110.

12. Kent $P$ T and Kubica G P. (1985): Public Health Mycobacteriology: A Guide for the Level III Labo.Centers for Disease Control, Atlanta, GA. Am. Respir. J., 161: 13761395.

13. Mohamed A , Hossam $M$ and Masoud $M$ E. (2019): Evaluation of GeneXpert as a new diagnostic tool for detection of pulmonary tuberculosis,The Egyptian Journal of Chest Diseases and Tuberculosis, 68: 270 -273.

14. Lawn SD and Nicol MP. (2011): Xpert (R) MTB/RIF assay: development, evaluation and implementation of a new rapid molecular diagnostic for tuberculosis and rifampicin resistance. Future Microbiol. J., 6 (9):10671082.

15. Lawn SD, Mwaba P, Bates M, Piatek A, Alexander H, Marais BJ, Cuevas LE, McHugh TD, Zijenah L, Kapata N, Abubakar I, McNerney R, Hoelscher M, Memish ZA, Migliori GB, Kim P, Maeurer $M$, Schito $M$ and Zumla A. (2013): Advances in tuberculosis diagnostics: the Xpert MTB/RIF assay and future prospects for a point-of-care test. Lancet Infect. Dis., 13(4): 349- 61.

16. Monika A, Ashish B, Vinay B and Sarjana D. (2016): Comparative Study of GeneXpert with ZN Stain and Culture in Samples of Suspected Pulmonary Tuberculosis, Clin.and Diag. Research J., 10 (5): DC09-DC12.
17. Pai M, Minion J, Sohn H, Zwerling A and Perkins MD. (2009): Novel and improved technologies for tuberculosis diagnosis: progress and challenges. J., Clin Chest Med., 30: 701-716.

18. Rasslan O, Hafez S F, Hashem M, Ahmed OI, Faramawy MA S, Khater W S, Saleh D A, Mohamed MI, Khalifa M A M, Shoukry F A and El-Moghazy E H. (2012): MODS assay in the diagnosis of multidrug-resistant tuberculosis.Int. J. Tuberc. Lung Dis., 16 (7): 941-6.

19. Sanogo M, Kone B, Diarra B, Maiga $M$, Baya B, Somboro AM, Sarro YS, Togo ACG, Dembele BPP, Goita D, Kone A, M'Baye O, Coulibaly N, Diabate S, Traore B, Diallo MH, Coulibaly YI, Saleeb P, Belson M, Orsega S, Siddiqui S, Polis MA, Dao S, Murphy RL and Diallo S. (2017): Performance of Microscopic Observation Drug Susceptibility for the Rapid Diagnosis of Tuberculosis and Detection of Drug Resistance in Bamako, Mali, Clin. Micro. Infect J., 3 (6): 408.e1-408.e6.

20. Sanchez PE, Merker $M$, Beckert $P$, Jochims F, Dlamini T, Kahn P, Bonnet M and Niemann S. (2015): Detection of drugresistant tuberculosis by Xpert MTB/RIF in Swaziland. N Engl, Med J., 372:1181 -1182.

21. Sharma $S$ K, Mikashmi $K$, Raj $N \mathbf{Y}$, Jigyasa $\mathbf{C}$, Dinkar B , Vishnubhatla $\mathrm{S}$, Rohini $S$ and Binit K. (2015): Evaluating the Diagnostic Accuracy of Xpert MTB/RIF Assay in Pulmonary Tuberculosis. Pone. J., 10:1371-1380.

22. WHO (2017): WHO Global Report. World Health Organization. Global tuberculosis report, WHO/HTM/TB/.13. Geneva.

23. WHO (2018): WHO. World Health Organization: Global Tuberculosis Report. Geneva: WHO Press.

24. Wilson M L. (2014): Recent advances in the laboratory detection of M.TB complex and drug Resistance,J. of Clin. Infect. Dis., 52 $1350-1355$. 


\section{تقبيم طريقتى الفحص المجهري و التفاعل الجينى التسلسلى في تشخيص الدرن الرئوى}

إيمان حسني محمد، عفت مصطفى حسن، فاتن على شكرى، أمنية أحمد الديدامونى

قسم الميكروبيولوجي، كلية الطب ( بنات )، جامعة الازهر

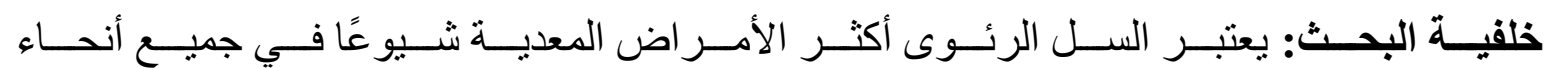

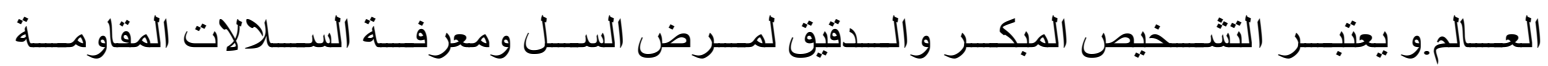
للأدوية ذو أهمية أساسية لكل من المرضى ومكافحة العدوى.

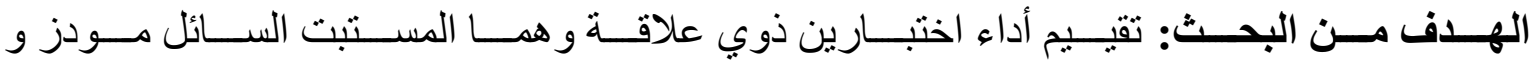

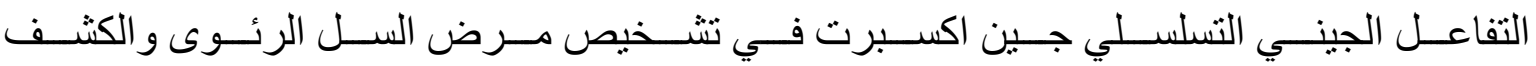

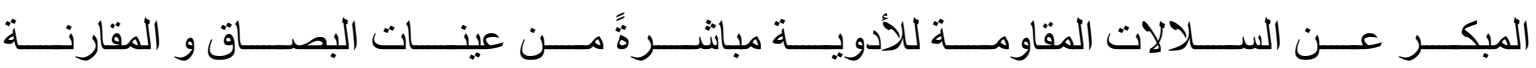

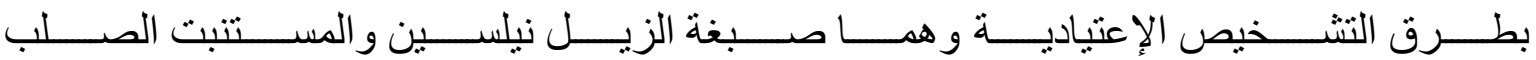
لوفنيشتاين جينسين.

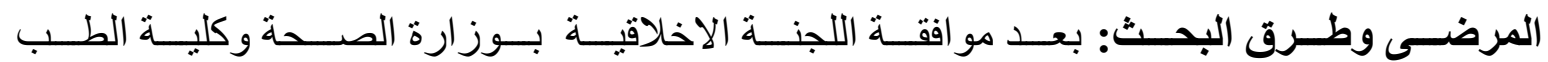

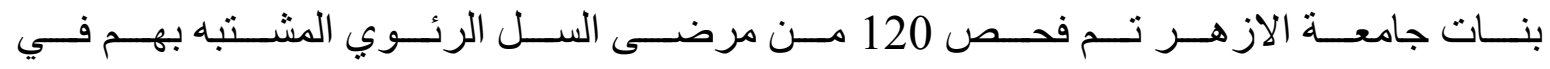

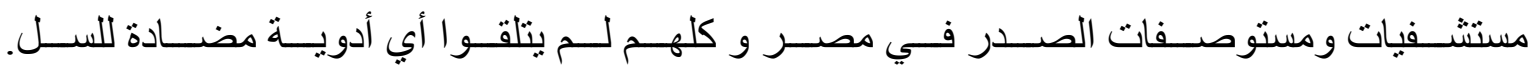

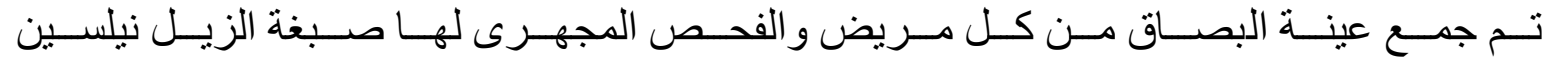

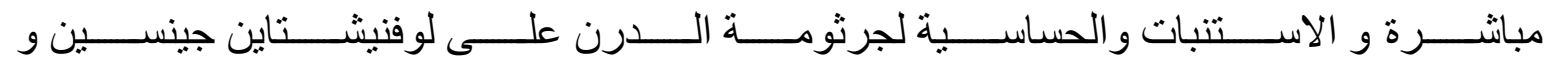

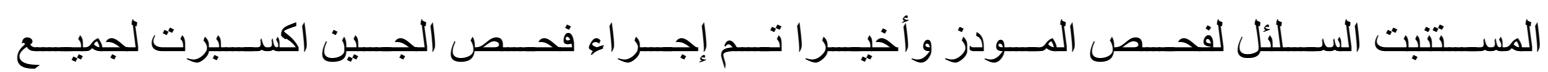

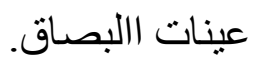

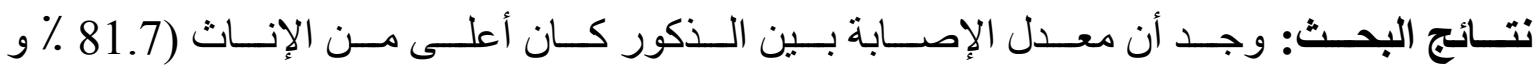

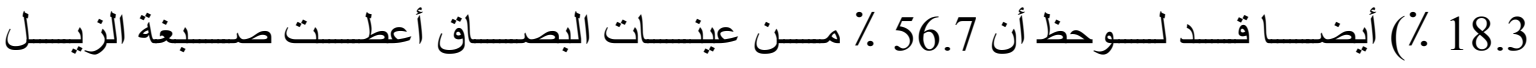

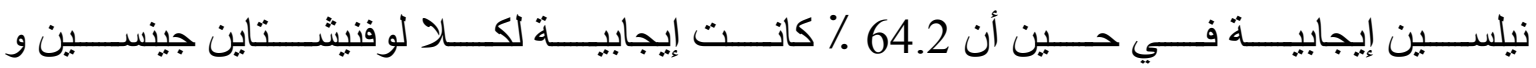

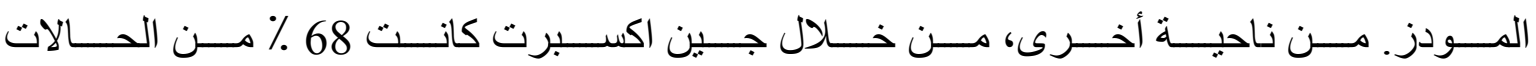

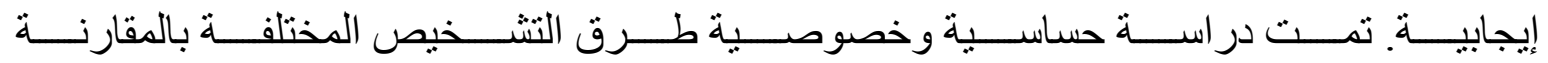

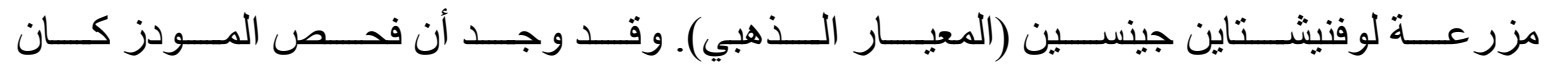

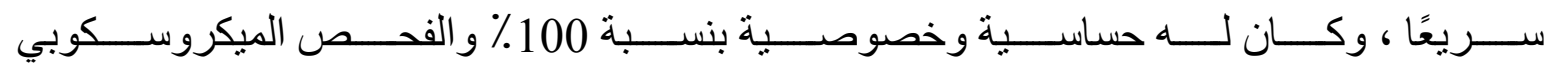




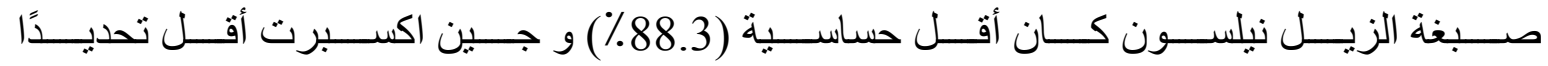

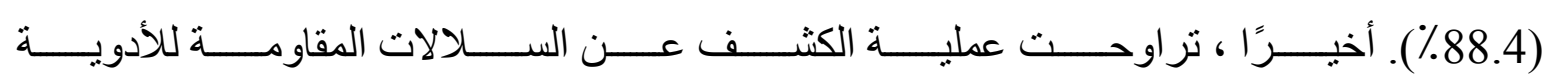

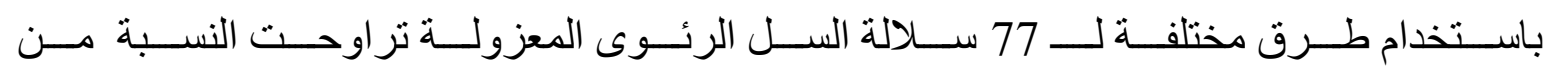

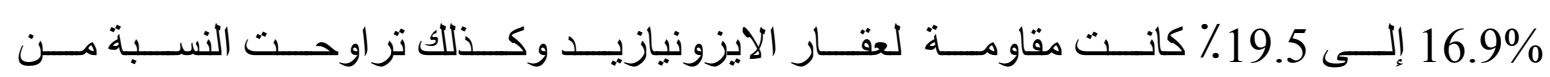

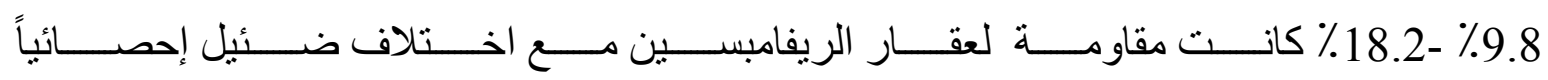

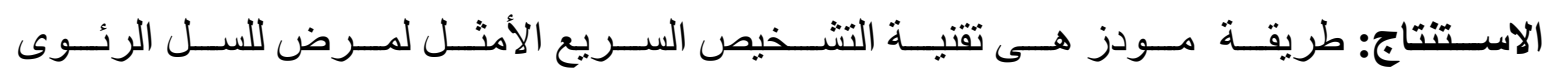

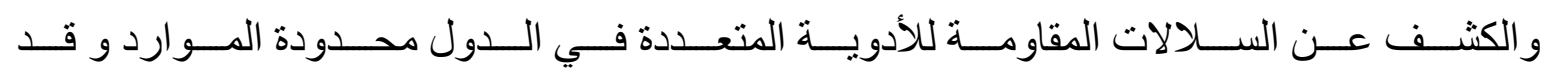

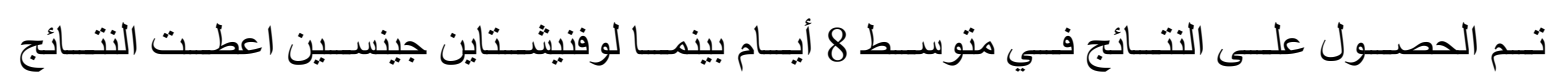

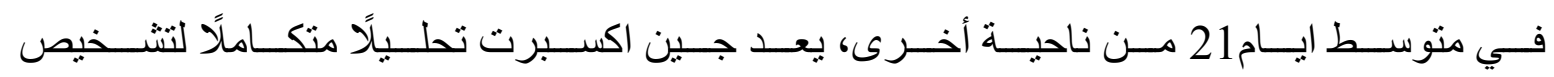

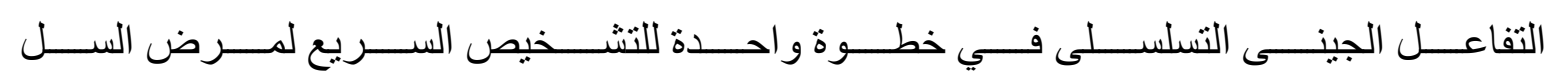
و الكثف عن السلالات المقاومة للريفامبسين خلال ساعتين ولكنه مكلف للغاية. 